\title{
Sliding Mode Control Based on Disturbance Observer for Greenhouse Climate Systems
}

\author{
Lijun Chen $(\mathbb{D}$, Shangfeng Du $(\mathbb{D}$, Dan Xu, Yaofeng He, and Meihui Liang \\ College of Information and Electrical Engineering, China Agricultural University, Beijing 100083, China \\ Correspondence should be addressed to Shangfeng Du; 13520760485@126.com
}

Received 25 July 2017; Revised 31 January 2018; Accepted 1 March 2018; Published 8 April 2018

Academic Editor: Xinkai Chen

Copyright (C) 2018 Lijun Chen et al. This is an open access article distributed under the Creative Commons Attribution License, which permits unrestricted use, distribution, and reproduction in any medium, provided the original work is properly cited.

\begin{abstract}
Greenhouses are closed environments that require careful climatic control, which can benefit from a system control method to cope with the high nonlinearity, complex coupling, and robustness of unknown disturbances. This paper presents a general framework for an integral sliding mode controller based on a disturbance observer combined with feedback linearization for a greenhouse temperature and humidity system. The first-principle greenhouse climate model is described as a standard affine nonlinear system. The feedback linearization control law is used to achieve a system consisting of two separate integrator channels for temperature and humidity. System compound disturbances are estimated by applying a sliding mode disturbance observer. Based on the observer, an integral sliding mode control is incorporated to enhance the robustness against uncertainties and guarantee satisfactory tracking performance even when there are unknown estimation errors. The validity and efficacy of the proposed control technique for greenhouse climate tracking were verified by comparison with simulation results obtained using the common sliding mode control method using feedback linearization without the disturbance observer. Based on this comparison, the developed controller shows a faster system response speed, higher control precision, and stronger anti-interference ability. This method can be applied to improve greenhouse climate control systems.
\end{abstract}

\section{Introduction}

Greenhouses constitute a closed environment in which climatic variables can be controlled to allow for the optimal growth and development of crops [1]. The creation of a favorable environment inside greenhouses requires the regulation of all relevant variables throughout plant development [2]. Over the past decades, the control design of the climatic conditions in greenhouses, notably temperature and humidity, has received considerable attention, and a wide variety of strategies have been represented. However, difficulties exist in achieving good control performance due to the highly coupled nonlinear dynamic behavior of such systems $[3,4]$. To address this problem, several control approaches have been developed by approximating the nonlinear model to a linear model. In particular, accurate feedback linearization (FL) approach based on geometric nonlinear control theory has attracted the attention of many researchers. In [5-8], this technique was successfully employed to linearize and decouple the complex greenhouse climate system, and the linearized system-based control method design was greatly simplified.

FL has proven to be an efficient control for nonlinear systems. However, FL must rely on a detailed dynamic model and is unable to cope with unknown changes in systems. It is difficult to obtain accurate knowledge of greenhouse climate systems, which usually suffer from the integrated effects of system uncertainty, unknown disturbances, and time-varying parameters $[9,10]$. Sliding mode control (SMC) is a powerful control method because of its strong robustness with respect to internal and external bounded disturbances, as well as parameter uncertainties [11]. He et al. [12] designed greenhouse climate robust control schemes by employing traditional sliding mode reaching laws. Luan et al. [13] combined a radial basis function neural network with SMC to cope with the problem of following varying desired climate trajectories in greenhouses. Although various greenhouse climate SMC schemes have been proposed, the dynamic information of the system disturbances has not been fully considered. SMC uses a switching control signal at a high 
frequency to enforce the system trajectories onto a surface [14]. This is the main reason why the approach has the attractive feature of keeping the system insensitive to uncertainties. Conversely, if these disturbance and uncertain terms cannot be effectively estimated, there will be severe chattering and poor dynamic behavior, hindering SMC from widespread application.

The integral sliding mode control (ISMC) strategy has been intensively studied to enhance robustness and reduce the influence of unknown disturbance terms that act on a closed-loop system. Oliveira et al. [15] applied the ISMC to the three-phase induction motor and achieved results of low overshoot and no steady-state error. To further enhance the antidisturbance ability of the system, the disturbance observer has been introduced for combination with ISMC to fully use the dynamic information of unknown disturbances. In particular, the sliding mode disturbance observer (SMDO) is a popular choice because of its independence on the detailed mathematical model of disturbances. This technique allows for model uncertainties to be computed without the use of high control gain or extensive computational power. Hall and Shtessel [16] proposed an SMDO-based control for a reusable launch vehicle. Besnard et al. [17] used a SMC driven by SMDO approach to design a robust flight controller for a small quadrotor vehicle and achieved the desired output tracking performance in the presence of the bounded disturbances. Chen et al. [18] estimated the unknown compound disturbance by developing a new SMDO to deal with the multi-input and multi-output nonlinear systems with uncertainty and dead-zone. The universal disturbance approximation capabilities of SMDO have made it an important choice for implementing in general-purpose control designs in the field of industry. However, it is rarely used for greenhouse climate control.

This paper provides a general framework for SMDObased adaptive ISMC of greenhouse temperature and humidity using an FL technique to cope with system nonlinearities, complex coupling, and modeling uncertainties and unknown disturbances in the process of tracking set points. The aim of this proposed overall control technique is to combine FL and ISMC driven by SMDO to realize their individual advantages. The use of FL simplifies and decouples the overall system model significantly. The ISMC is able to reduce the chattering and improve the transient response due to the implementation of SMDO and possesses the desired characteristics of robustness and good performance. The organization of the paper is as follows. Section 2 describes the greenhouse dynamics physical model. Section 3 provides a discussion of the control system structure. The closed-loop system stability is proven using the Lyapunov method. Section 4 shows the results of several simulations to evaluate the performance of the proposed technique. Finally, conclusions are drawn in Section 5 .

\section{Physical Model of Greenhouse Dynamics}

Greenhouse climates are a combination of physical processes involving energy transfer and mass balance, which are generated from the differences between inside and outside climatic conditions and the effect of the actuators $[19,20]$. For simplicity, the air and mass in the greenhouse is considered to have a homogenous distribution. The temperature and humidity model based on energy and mass conversation is described as follows [21]:

$$
\begin{aligned}
& \frac{d T_{\text {in }}(t)}{d t} \\
& =\frac{1}{\rho C_{g} V_{T}}\left(Q_{\text {heat }}(t)+S_{r}(t)-\lambda Q_{\text {fog }}(t)\right) \\
& \quad-\frac{V_{R}(t)}{V_{T}}\left(T_{\text {in }}(t)-T_{\text {out }}(t)\right) \\
& \quad-\frac{U_{A}}{\rho C_{g} V_{T}}\left(T_{\text {in }}(t)-T_{\text {out }}(t)\right), \\
& \frac{d w_{\text {in }}(t)}{d t} \quad \frac{1}{V_{H}} Q_{\text {fog }}(t)+\frac{1}{V_{H}}\left(E\left(S_{r}(t), w_{\text {in }}(t)\right)\right) \\
& \quad-\frac{V_{R}(t)}{V_{H}}\left(w_{\text {in }}(t)-w_{\text {out }}(t)\right), \\
& E\left(S_{r}(t), w_{\text {in }}(t)\right)=\alpha \frac{S_{r}(t)}{\lambda}-\beta w_{\text {in }}(t),
\end{aligned}
$$

where $T_{\text {in }}\left({ }^{\circ} \mathrm{C}\right)$ and $T_{\text {out }}\left({ }^{\circ} \mathrm{C}\right)$ are the temperatures inside and outside the greenhouse, respectively, $\rho\left(1.2 \mathrm{~kg} / \mathrm{m}^{3}\right)$ is the air density, $C_{g}(1006 \mathrm{~J} /(\mathrm{kg} \cdot \mathrm{K}))$ is the specific heat of air, $Q_{\text {heat }}$ $(\mathrm{W})$ is the input heating provided by the greenhouse heater, $S_{r}(\mathrm{~W})$ is the intercepted solar radiant energy, $\lambda(2257 \mathrm{~J} / \mathrm{g})$ is the latent heat of vaporization, $Q_{\mathrm{fog}}\left(\mathrm{g} \mathrm{H}_{2} \mathrm{O} / \mathrm{s}\right)$ is the water capacity of the fog system, $V_{R}\left(\mathrm{~m}^{3} / \mathrm{s}\right)$ is the ventilation rate, $U_{A}(\mathrm{~W} / \mathrm{K})$ is the heat transfer coefficient of enclosure, $w_{\text {in }}$ ( $\mathrm{g}$ $\left.\mathrm{H}_{2} \mathrm{O} / \mathrm{m}^{3}\right)$ and $w_{\text {out }}\left(\mathrm{g} \mathrm{H}_{2} \mathrm{O} / \mathrm{m}^{3}\right)$ are the inside and outside absolute humidities, respectively, $V_{T}\left(\mathrm{~m}^{3}\right)$ and $V_{H}\left(\mathrm{~m}^{3}\right)$ are the temperature and humidity active mixing air volumes, respectively, $E\left(\mathrm{~g} \mathrm{H}_{2} \mathrm{O} / \mathrm{s}\right)$ is the evapotranspiration rate of the crop leaf, $\alpha$ is the shading and leaf area index coefficient, and $\beta$ is the coefficient for thermodynamic constants and other factors affecting evapotranspiration.

Only operation during summer is considered herein; therefore, $Q_{\text {heat }}$ in (1) is set to zero, and the term $\beta w_{\text {in }}$ can be neglected due to the fact that the ventilation-cooling operating conditions are dominated by solar radiation alone [22]. We define $C_{0}=\left(\rho C_{g} V_{T}\right)$ and $\alpha_{0}=\alpha\left(\lambda V_{H}\right)^{-1}$ and normalize the control variables by using the convention that $V_{R, \%}=V_{R} / V_{R, \text { max }}, Q_{\text {fog, } \%}=Q_{\text {fog }} / Q_{\text {fog, } \max }, \lambda_{0}=\lambda Q_{\text {fog, } \max }$, and $V_{0}=V_{H} / Q_{\text {fog,max }}$, in which $V_{R, \max }$ represents the maximum ventilation rate and $Q_{\text {fog,max }}$ represents the maximum capacity of the fog system. Then, models (1)-(3) can be rewritten in the following simpler form [22]:

$$
\frac{d T_{\mathrm{in}}(t)}{d t}=\frac{1}{C_{0}}\left(S_{r}(t)-\lambda_{0} Q_{\mathrm{fog}, \%}(t)\right)
$$




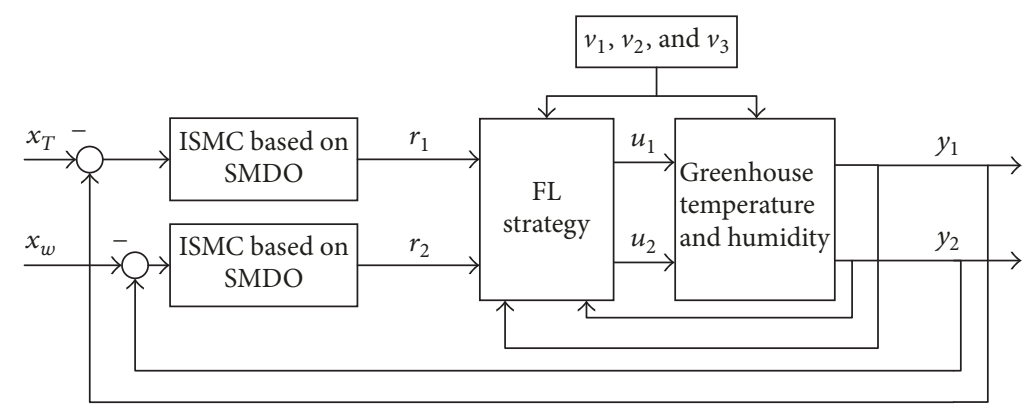

FIGURE 1: Schematic diagram of the control system structure for the entire greenhouse climate.

$$
\begin{aligned}
& -\frac{V_{R, \%}(t)}{t_{v}}\left(T_{\text {in }}(t)-T_{\text {out }}(t)\right) \\
& -\frac{U_{A}}{C_{0}}\left(T_{\text {in }}(t)-T_{\text {out }}(t)\right), \\
\frac{d w_{\text {in }}(t)}{d t}= & \frac{1}{V_{0}} Q_{\text {fog }, \%}(t)+\alpha_{0} S_{r}(t) \\
& -\frac{V_{R, \%}(t)}{t_{v}}\left(w_{\text {in }}(t)-w_{\text {out }}(t)\right),
\end{aligned}
$$

where $t_{v}$ represents the inverse of the number of air changes per unit time.

\section{Greenhouse Climate System Controller Design}

3.1. Feedback Linearization Strategy. As shown in (4), the greenhouse climate system is characterized by high nonlinearity and coupling, but it is linear to the control inputs. Defining the state variable $x=\left[x_{1}, x_{2}\right]^{T}=\left[T_{\text {in }}, w_{\text {in }}\right]^{T}$, control variable $u=\left[u_{1}, u_{2}\right]^{T}=\left[V_{R, \%}, Q_{\text {fog, } \%}\right]^{T}$, and outside disturbance variable $v=\left[v_{1}, v_{2}, v_{3}\right]^{T}=\left[S_{r}, T_{\text {out }}, w_{\text {out }}\right]^{T}$ can yield the inside temperature and humidity system in a normal form of a multiple-input, multiple-output affine nonlinear system, as shown in the following:

$$
\begin{aligned}
& \dot{x}=F(x, v)+G(x, v) u, \\
& y=x,
\end{aligned}
$$

where $y$ is the system output, $F(x, v)$ is the state function vector, and $G(x, v)$ is the control gain matrix, which are given as

$$
\begin{aligned}
& F(x, v)=\left[\begin{array}{l}
F_{1}(x, v) \\
F_{2}(x, v)
\end{array}\right]=\left[\begin{array}{c}
-\frac{U_{A}}{C_{0}} x_{1}+\frac{1}{C_{0}} v_{1}+\frac{U_{A}}{C_{0}} v_{2} \\
\alpha_{0} v_{1}
\end{array}\right], \\
& G(x, v)=\left[\begin{array}{l}
G_{1}(x, v) \\
G_{2}(x, v)
\end{array}\right]=\left[\begin{array}{cc}
-\frac{1}{t_{v}}\left(x_{1}-v_{2}\right) & -\frac{\lambda_{0}}{C_{0}} \\
-\frac{1}{t_{v}}\left(x_{2}-v_{3}\right) & \frac{1}{V_{0}}
\end{array}\right] .
\end{aligned}
$$

Assuming that $G(x, v)$ is nonlinear, applying the $\mathrm{FL}$ method results in the following integrator system:

$$
\dot{x}=r \text {, }
$$

where $r=\left[r_{1}, r_{2}\right]^{T}$ is the new control input vector in the transformed coordinate system. Finally, the FL control law takes the following form:

$$
u=G^{-1}(x, v)[-F(x, v)+r] .
$$

\subsection{Adaptive Sliding Mode Control Based on Disturbance} Observer. The dynamic model of energy and mass balance of greenhouse climate is shown to be linearized accurately and decoupled absolutely by FL. The equivalent system consists of two independent integrator channels for temperature and humidity, which makes it suitable for designing adaptive ISMC based on SMDO separately. The control system structure for the entire greenhouse climate is illustrated in Figure 1, where $x_{T}$ and $x_{w}$ are the desired temperatureand humidity-tracking trajectories, respectively. The control structure behaves as two decoupled control loops. The controller design process is the same for greenhouse temperature and humidity channels. The ISMC based on SMDO has been derived in its continuous form by Chen et al. [18]. This approach has been applied successfully to the attitude control of the near space vehicle. In the present study, using the temperature controller design as an example, the algorithm is briefly presented based on the work of the authors.

3.2.1. Designing of Sliding Mode Disturbance Observer. The FL technique will perform properly if the nonlinear functions $F(x, v)$ and $G(x, v)$ are known precisely. However, in practice, $F(x, v)$ and $G(x, v)$ are often only known with uncertainty and there are other unknown time-varying disturbances in the system. In this study, the SMDO is developed to approximate the unknown compound disturbance of the equivalent system (7).

The uncertain linearized greenhouse temperature system is considered based on (5) and (7), as follows:

$$
\dot{x}_{1}=r_{1}+\Delta F_{1}\left(x_{1}, v\right)+\Delta G_{1}\left(x_{1}, v\right) u_{1}+d_{1},
$$

where $\Delta F_{1}\left(x_{1}, v\right)$ and $\Delta G_{1}\left(x_{1}, v\right)$ are the continuous system uncertainties and $d_{1}$ is the unknown time-varying disturbance. It is reasonable to assume that $\Delta F_{1}\left(x_{1}, v\right), \Delta G_{1}\left(x_{1}, v\right)$, 
and $d_{1}$ are all bounded. For convenience, the bounded compound disturbance is defined as $D_{1}\left(x_{1}, v\right)=\Delta F_{1}\left(x_{1}, v\right)+$ $\Delta G_{1}\left(x_{1}, v\right) u_{1}+d_{1}$. Then, it is assumed that an unknown positive constant exists, $\theta$, such that $\left|\dot{D}_{1}\right| \leq \theta$. obtain

Let the tracking error be $e_{1}=x_{1}-x_{T}$. Invoking (9), we

$$
\dot{e}_{1}=r_{1}+D_{1}\left(x_{1}, v\right)-\dot{x}_{T} \text {. }
$$

The SMDO is designed as follows [18]:

$$
\begin{aligned}
\sigma_{0} & =z-e_{1}, \\
\dot{z} & =r_{1}+\widehat{D}_{1}-\dot{x}_{T}, \\
\sigma_{1} & =\sigma_{0}+L \dot{\sigma}_{0}, \\
\dot{\widehat{D}}_{1} & =-L^{-1}\left(\dot{\sigma}_{0}+a_{1} \sigma_{1}+a_{2} \operatorname{sgn}\left(\sigma_{1}\right)+\widehat{a}_{3} \operatorname{sgn}\left(\sigma_{1}\right)\right),
\end{aligned}
$$

where $\sigma_{0}, \sigma_{1}$, and $z$ are the auxiliary variables, $\widehat{D}_{1}$ is the estimated value of $D_{1}$, and $L>0, a_{1}>0$, and $a_{2}>0$ are the parameters to be designed. $L$ is designed to satisfy the Hurwitz stability theorem. The parameters $a_{1}$ and $a_{2}$ affect the system chattering and the convergence speed. Generally, the larger $a_{1}$ and $a_{2}$ can result in higher response speed and stronger chattering, and vice versa. In practice, the parameters can be chosen based on the designers' demand for the observer output performance. $\widehat{a}_{3}$ is the estimate of $a_{3}$ with $a_{3}$ being the maximum of $L \theta$ and $\operatorname{sgn}(\cdot)$ is the sign function. Note that the differential term $\dot{\sigma}_{0}$ can be calculated using the well-known, practical, real-time sliding mode differentiator [23].

The adaptive parameter updated law of $\widehat{a}_{3}$ is given as follows:

$$
\dot{\hat{a}}_{3}=\gamma_{0}\left|\sigma_{1}\right|
$$

where $\gamma_{0}>0$ is the constant to be designed.

The derivatives of $\sigma_{0}$ and $\sigma_{1}$ are formulated as follows:

$$
\begin{aligned}
\dot{\sigma}_{0}= & \dot{z}-\dot{e}_{1}=r_{1}+\widehat{D}_{1}-\dot{x}_{T}-\left(r_{1}+D_{1}-\dot{x}_{T}\right) \\
= & \widehat{D}_{1}-D_{1}=-\widetilde{D}_{1}, \\
\dot{\sigma}_{1}= & \dot{\sigma}_{0}+L \ddot{\sigma}_{0}=\dot{\sigma}_{0}+L\left(\dot{\vec{D}}_{1}-\dot{D}_{1}\right) \\
= & \dot{\sigma}_{0}-\left(\dot{\sigma}_{0}+a_{1} \sigma_{1}+a_{2} \operatorname{sgn}\left(\sigma_{1}\right)+\widehat{a}_{3} \operatorname{sgn}\left(\sigma_{1}\right)\right) \\
& -L \dot{D}_{1} \\
= & -\left(a_{1} \sigma_{1}+a_{2} \operatorname{sgn}\left(\sigma_{1}\right)+\widehat{a}_{3} \operatorname{sgn}\left(\sigma_{1}\right)+L \dot{D}_{1}\right) .
\end{aligned}
$$

To analyze the stability of the disturbance approximation error $\widetilde{D}_{1}$ and the parameter estimate error $\widetilde{a}_{3}$ with $\widetilde{a}_{3}=a_{3}-$ $\widehat{a}_{3}$, choose the Lyapunov function as follows:

$$
V_{0}=\frac{1}{2} \sigma_{1}^{2}+\frac{1}{2 \gamma_{0}} \widetilde{a}_{3}^{2}
$$

Considering (12)-(14) and differentiating $V_{0}$, we obtain

$$
\begin{aligned}
\dot{V}_{0} & =\sigma_{1} \dot{\sigma}_{1}+\frac{1}{\gamma_{0}} \widetilde{a}_{3} \dot{\tilde{a}}_{3} \\
& =-a_{1} \sigma_{1}^{2}-a_{2}\left|\sigma_{1}\right|-\widehat{a}_{3}\left|\sigma_{1}\right|-\sigma_{1} L \dot{D}_{1}+\frac{1}{\gamma_{0}} \widetilde{a}_{3} \dot{\hat{a}}_{3} \\
& \leq-a_{1} \sigma_{1}^{2}-a_{2}\left|\sigma_{1}\right|-\widehat{a}_{3}\left|\sigma_{1}\right|+a_{3}\left|\sigma_{1}\right|+\frac{1}{\gamma_{0}} \widetilde{a}_{3} \dot{\hat{a}}_{3} \\
& =-a_{1} \sigma_{1}^{2}-a_{2}\left|\sigma_{1}\right|+\widetilde{a}_{3}\left|\sigma_{1}\right|+\frac{1}{\gamma_{0}} \widetilde{a}_{3} \dot{\hat{a}}_{3} \\
& =-a_{1} \sigma_{1}^{2}-a_{2}\left|\sigma_{1}\right| .
\end{aligned}
$$

The applied disturbance observer (11) and adaptive parameter updated law (12) can guarantee that the disturbance estimated error reaches zero in a limited time [18]. It is worth noting that the designed SMDO only requires the bounded derivative of the unknown disturbance to ensure the asymptotic convergence of the disturbance estimate error. Compared with other SMDOs reported in the literature with the requirement of providing the upper boundary of the disturbance, the restrictive condition imposed on the disturbance is relaxed.

3.2.2. Designing of Integral Sliding Mode Controller. To alleviate chattering and improve the control performance, the adaptive ISMC using the disturbance estimated value based on the SMDO is developed. The problem is to design continuous control $r_{1}$ that provides asymptotic output tracking as time increases in the presence of bounded disturbances. First, the switching surface is defined, which should be designed to provide optimal system performance and satisfy the desired control purposes [24]. The sliding surface is usually defined by a linear combination of state variables error and its derivatives. Considering that the state variable number is one, to cope with the tracking error and disturbance approximation error, the sliding surface of the ISMC is defined as follows:

$$
s=a_{4} e_{1}+a_{4} \sigma_{0}+\int_{0}^{t} a_{5} e_{1} d t
$$

where $a_{4}$ and $a_{5}$ are positive constants with values that need to be designed. $a_{4}$ and $a_{5}$ decide the dynamic behavior of the final sliding mode. They can be chosen by ways of pole assignment, optimal method, and Lyapunov function, among others. Here, $a_{4}$ and $a_{5}$ are determined by using Hurwitz theorem to maintain stability condition of the sliding mode. With respect to the integral sliding mode surface, the condition that the system is in sliding mode at the initial time is required.

To obtain the control signal $r_{1}$, the sliding surface and its derivative must be considered as zero. By performing this, the steady-state error will approach zero. Substituting the exponential reaching law $\dot{s}=-k s-\varepsilon \operatorname{sgn}(s)$ into (17) generates the control law:

$$
r_{1}=-a_{4}^{-1}\left(k s+\varepsilon \operatorname{sgn}(s)+a_{4} \widehat{D}_{1}-a_{4} \dot{x}_{T}+a_{5} e_{1}\right),
$$




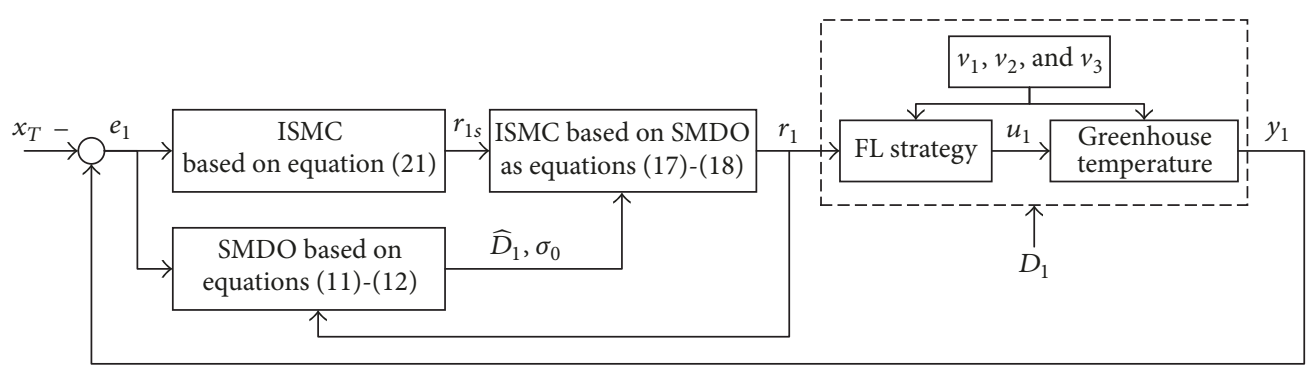

FIGURE 2: The structure of the ISMC based SMDO.

where $k>0$ and $\varepsilon>0$ are the control parameters to be designed. The value of $k$ has an effect on the speed when the system approaches the sliding mode. The larger $k$ can accelerate the regulation process and convergence velocity. $\varepsilon$ represents the approaching speed near the switching surface. The larger $\varepsilon$, the smaller the chattering and the lower the approaching speed, allowing the controller towards a smoother behavior, and vice visa. There is need to take overshoot and settling time into consideration when regulating $\varepsilon$.

To check the stability of the system tracking error, the positive definite Lyapunov function is considered as follows:

$$
V_{1}=\frac{1}{2} s^{2}
$$

Considering (10), (13), and (17)-(18) and differentiating $V_{1}$, we obtain

$$
\begin{aligned}
\dot{V}_{1} & =s \dot{s}=s\left(a_{4} \dot{e}_{1}+a_{4} \dot{\sigma}_{0}+a_{5} e_{1}\right) \\
& =s\left(a_{4}\left(r_{1}+D_{1}-\dot{x}_{T}\right)-a_{4} \widetilde{D}_{1}+a_{5} e_{1}\right)=s\left(a_{4} r_{1}\right. \\
& \left.+a_{4} \widehat{D}_{1}-a_{4} \dot{x}_{T}+a_{5} e_{1}\right)=s(-k s-\varepsilon \operatorname{sgn}(s) \\
& \left.-a_{4} \widehat{D}_{1}+a_{4} \dot{x}_{T}-a_{5} e_{1}+a_{4} \widehat{D}_{1}-a_{4} \dot{x}_{T}+a_{5} e_{1}\right) \\
& =-k s^{2}-\varepsilon|s| .
\end{aligned}
$$

If coefficients $k$ and $\varepsilon$ are selected as positive, $\dot{V}_{1}$ must always be negative to maintain the stability of the Lyapunov theorem. The negative $\dot{V}_{1}$ shows that, under the control law of (18), the sliding mode exists and can be reached. According to (16) and (20), it can be deduced that $\dot{V}=\dot{V}_{0}+\dot{V}_{1}$ is negative and that the applied control signal can guarantee that the system tracking error approaches zero asymptotically. In all, the designed overall control technique can guarantee the robustness, stability, and convergence of the closed-loop system.

Figure 2 shows the control structure of the ISMC based on SMDO for temperature system. It can be seen that the proposed algorithm combines the classical ISMC and the SMDO. The control law of the classical ISMC without the SMDO is defined as follows:

$$
\begin{aligned}
s & =a_{4} e_{1}+\int_{0}^{t} a_{5} e_{1} d t, \\
r_{1 s} & =-a_{4}^{-1}\left(k s+\varepsilon \operatorname{sgn}(s)-a_{4} \dot{x}_{T}+a_{5} e_{1}\right) .
\end{aligned}
$$

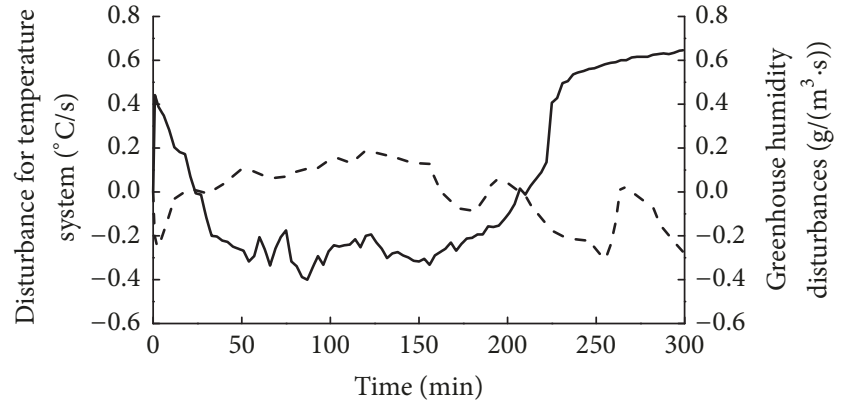

- Disturbance for temperature system
--- Disturbance for humidity system

FIgURE 3: Disturbances for temperature and humidity system.

The SMDO is used to evaluate the disturbance and is designed as (11). The overall control technique to calculate the control input $r_{1}$ can be derived from (18).

\section{Simulation and Results}

To evaluate the efficiency and performance of the proposed ISMC strategy using the SMDO based on the FL control law, which depends on physical models (4), a set point tracking simulation experiment was performed. For the simulation, the total simulation time was $300 \mathrm{~min}$. The initial values of the greenhouse temperature and humidity were $30^{\circ} \mathrm{C}$ and $18 \mathrm{~g} / \mathrm{m}^{3}$, respectively. The model parameters described by (4) were set as $C_{0}=-324.67 \mathrm{~min} \cdot \mathrm{W} /{ }^{\circ} \mathrm{C}, U_{A}=29.8 \mathrm{~W} /{ }^{\circ} \mathrm{C}$, $t_{v}=3.41 \mathrm{~min}, \lambda_{0}=465 \mathrm{~W}, \alpha_{0}=0.0033 \mathrm{~g} /\left(\mathrm{m}^{3} \cdot \mathrm{min} \cdot \mathrm{W}\right)$, and $V_{0}^{-1}=13.3 \mathrm{~g} /\left(\mathrm{m}^{3} \cdot \mathrm{min}\right)$, and they were expressed per square meter of greenhouse area [22]. The outside weather conditions were assumed to be $S_{r}=300 \mathrm{~W} / \mathrm{m}^{2}, T_{\text {out }}=$ $35^{\circ} \mathrm{C}$, and $w_{\text {out }}=4 \mathrm{~g} / \mathrm{m}^{3}$. The temperature set point remained constant at $30^{\circ} \mathrm{C}$ until $100 \mathrm{~min}$, when it changed to $26^{\circ} \mathrm{C}$. At $200 \mathrm{~min}$, the humidity set point changed from 18 to $24 \mathrm{~g} / \mathrm{m}^{3}$. It was assumed that the greenhouse climate system integrated unknown time-varying disturbances varied on the time scale of minutes and had a correlation with the outside weather conditions variations. As the outdoor solar radiation had an important effect on the indoor temperature and the outdoor humidity on the indoor humidity, the disturbances for temperature and humidity system were set related to these two outdoor variables, respectively. Figure 3 shows the disturbances. 


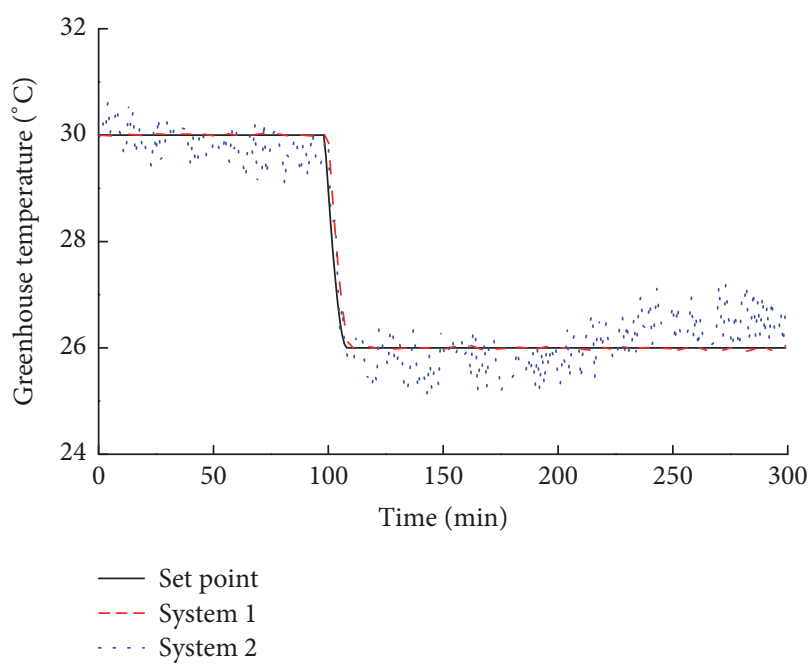

(a)

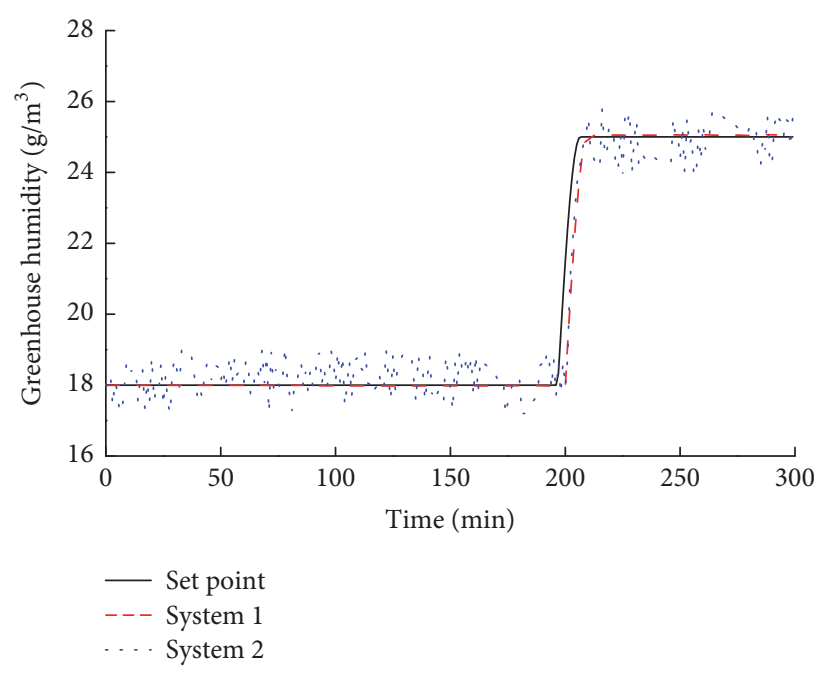

(b)

FIgURE 4: Comparison of tracking results between systems 1 and 2 for (a) temperature and (b) humidity.

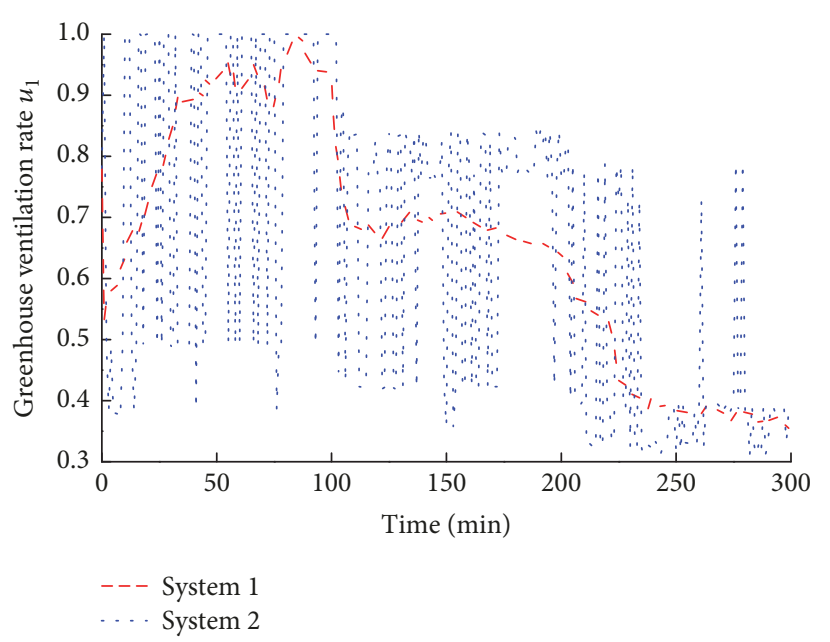

(a)

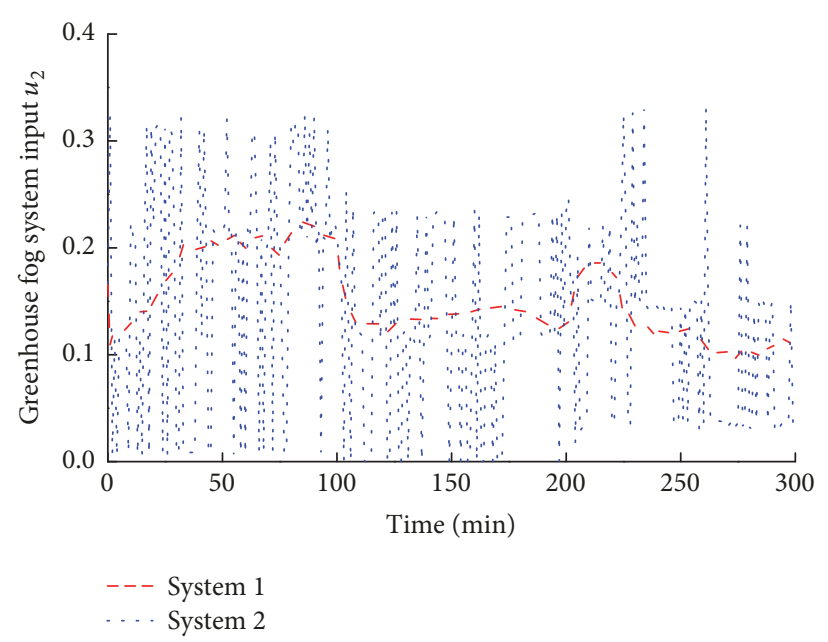

(b)

Figure 5: Comparison of the control inputs of systems 1 and 2 for (a) normalized ventilation rate variable and (b) normalized fog system input variable.

According to the aforementioned parameters regulation method, after a series of simulation debugging, to get a good control performance, the parameters of ISMC driven by SMDO for temperature were chosen as $L=0.5, a_{1}=0.02$, $a_{2}=0.03, \gamma_{0}=0.05, a_{4}=100, a_{5}=0.01, k=0.08$, and $\varepsilon=0.8$. The parameters of ISMC driven by SMDO for humidity were chosen as $L=1, a_{1}=0.01, a_{2}=0.01, \gamma_{0}=0.02$, $a_{4}=100, a_{5}=0.008, k=0.5$, and $\varepsilon=2$.

To further demonstrate the control performance of the developed control strategy, it was compared with an ISMC based on FL without using an SMDO. For convenience, the approaches with and without SMDO are denoted as systems 1 and 2, respectively. The control law in system 2 is defined as (21). The parameters selection strategy is the same as system 1. By some tests, the parameters were chosen as $a_{4}=1, a_{5}=$ $0.01, k=0.02$, and $\varepsilon=0.65$ for the temperature controller design and $a_{4}=1, a_{5}=0.008, k=0.09$, and $\varepsilon=0.95$ for the humidity controller design.

The obtained simulation comparison responses of the two systems are shown in Figures 4 and 5. Figure 4 presents the tracking results of the inside temperature and humidity. Both controllers were able to regulate the greenhouse climate around the given set point while rejecting disturbances. However, it costs less time for system 1 to reach the steady state, and chattering was clearly alleviated in system 1 . There was severe chattering in system 2 due to the inability to observe the disturbances online. System 1 achieved better static and dynamic tracking performance than system 2 . Figure 5 presents the compared control inputs for greenhouse climate. The control inputs of the two systems exhibited the same variation trend. However, the values of the control variables of system 1 were more stable than those of system 2 . 


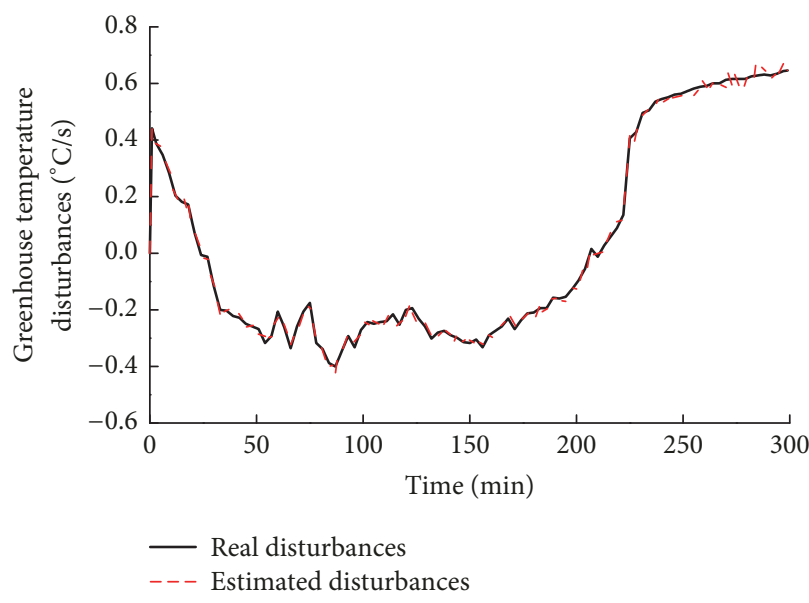

(a)

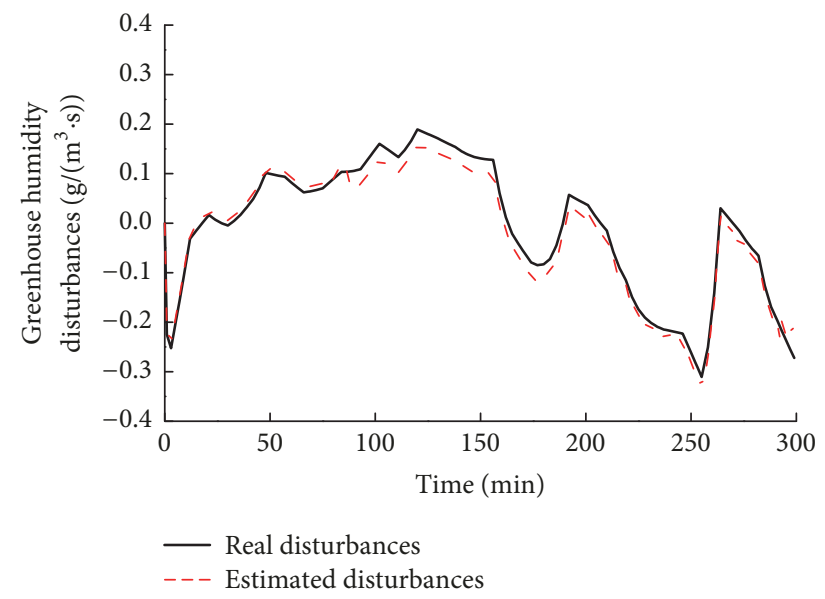

(b)

FIgURE 6: Comparison of real disturbances and estimated disturbances by system 1 for (a) temperature and (b) humidity.

Figure 6 illustrates a comparison between the real disturbances and estimated values by system 1 . There were small errors between the estimated and real disturbances and both the SMDOs for temperature and humidity worked well. The overall comparison simulation results indicate that the developed technique can offer more suitable control variables and enhance the tracking control performance under the integrated effect of unknown external disturbance. The reasons for this superiority are the use of SMDO to accurately estimate the time-varying disturbance.

\section{Conclusions}

A FL-based ISMC using a SMDO technique for greenhouse climate systems is proposed in this paper. FL can be applied to linearize and decouple greenhouse climate systems and simplify the SMDO and ISMC design based on the achieved integrator system. To facilitate the use of the FL without prior knowledge of the greenhouse climate dynamics, the ISMC driven by SMDO is used to strengthen the robustness to external disturbance and model uncertainty, enabling the FL technique to be used in an adaptive manner. The whole method can guarantee uniformly stable adaptation and asymptotic tracking even if there are inherent disturbance estimated errors. Finally, the results of the proposed method in this paper and a standard ISMC using FL without the SMDO are compared in terms of chattering magnitude, steady-state error, and dynamic performance. The results show that the developed technique offers adequate control performance in regulating greenhouse climate around the desired reference in the presence of system uncertainty and external disturbance. The proposed method is expected to gain wide acceptance in modern climate control systems. In future work, the described study will be applied in practical implementation to evaluate the system effectiveness.

\section{Conflicts of Interest}

The authors declare that there are no conflicts of interest regarding the publication of this article.

\section{Acknowledgments}

This work has been supported by the National Natural Science Foundation of China (Grant nos. 61174088 and 60374030).

\section{References}

[1] F. Rodríguez, J. L. Guzmán, M. Berenguel, and M. R. Arahal, "Adaptive hierarchical control of greenhouse crop production," International Journal of Adaptive Control and Signal Processing, vol. 22, no. 2, pp. 180-197, 2008.

[2] N. Bennis, J. Duplaix, G. Enéa, M. Haloua, and H. Youlal, "Greenhouse climate modelling and robust control," Computers and Electronics in Agriculture, vol. 61, no. 2, pp. 96-107, 2008.

[3] M. Azaza, K. Echaieb, F. Tadeo, E. Fabrizio, A. Iqbal, and A. Mami, "Fuzzy Decoupling Control of Greenhouse Climate," Arabian Journal for Science and Engineering, vol. 40, no. 9, pp. 2805-2812, 2015.

[4] P. J. M. van Beveren, J. Bontsema, G. van Straten, and E. J. van Henten, "Optimal control of greenhouse climate using minimal energy and grower defined bounds," Applied Energy, vol. 159, pp. 509-519, 2015.

[5] E. H. Gurban, T.-L. Dragomir, and G.-D. Andreescu, "Greenhouse climate control enhancement by using genetic algorithms," Control Engineering and Applied Informatics, vol. 16, no. 3, pp. 35-45, 2014.

[6] E. H. Gurban and G.-D. Andreescu, "Comparison study of PID controller tuning for greenhouse climate with feedback-feedforward linearization and decoupling," in Proceedings of the 2012 16th International Conference on System Theory, Control and Computing, ICSTCC 2012, rou, October 2012.

[7] I. A. Hameed and C. G. Sorensen, "A more energy efficient controller for the greenhouses climate control system," Applied Engineering in Agriculture, vol. 26, no. 3, pp. 491-498, 2010.

[8] S. Piñón, E. F. Camacho, B. Kuchen, and M. Peña, "Constrained predictive control of a greenhouse," Computers and Electronics in Agriculture, vol. 49, no. 3, pp. 317-329, 2005.

[9] J. L. Tovany, R. Ross-León, J. Ruiz-León, A. Ramírez-Treviño, and O. Begovich, "Greenhouse modeling using continuous 
timed Petri nets," Mathematical Problems in Engineering, vol. 2013, Article ID 639306, 9 pages, 2013.

[10] A. Maher, E. Kamel, F. Enrico, I. Atif, and M. Abdelkader, "An intelligent system for the climate control and energy savings in agricultural greenhouses," Energy Efficiency, vol. 9, no. 6, pp. 1241-1255, 2016.

[11] O. Lopez-Santos, L. Martinez-Salamero, G. Garcia, H. Valderrama-Blavi, and T. Sierra-Polanco, "Robust sliding-mode control design for a voltage regulated quadratic boost converter," IEEE Transactions on Power Electronics, vol. 30, no. 4, pp. 23132327, 2015.

[12] N. He, G. Yi, and S. Liang, "Tracking time-discrete quasi-sliding mode variable structure controller for temperature and humidity of greenhouse," in Proceedings of the 12th IEEE International Conference on Mechatronics and Automation, ICMA 2015, pp. 2273-2278, China, August 2015.

[13] X. Luan, P. Shi, and F. Liu, "Robust adaptive control for greenhouse climate using neural networks," International Journal of Robust and Nonlinear Control, vol. 21, no. 7, pp. 815-826, 2011.

[14] Y. Xia, Z. Zhu, C. Li, H. Yang, and Q. Zhu, "Robust adaptive sliding mode control for uncertain discrete-time systems with time delay," Journal of The Franklin Institute, vol. 347, no. 1, pp. 339-357, 2010.

[15] C. M. R. Oliveira, M. L. Aguiar, J. R. B. A. Monteiro, W. C. A. Pereira, G. T. Paula, and T. E. P. Almeida, "Vector control of induction motor using an integral sliding mode controller with anti-windup," Journal of Control Automation \& Electrical Systems, vol. 27, no. 2, pp. 169-178, 2016.

[16] C. E. Hall and Y. B. Shtessel, "Sliding mode disturbance observer-based control for a reusable launch vehicle," Journal of Guidance Control \& Dynamics, vol. 29, no. 6, pp. 1315-1328, 2006.

[17] L. c. Besnard, Y. B. Shtessel, and B. Landrum, "Quadrotor vehicle control via sliding mode controller driven by sliding mode disturbance observer," Journal of The Franklin Institute, vol. 349, no. 2, pp. 658-684, 2012.

[18] M. Chen, S.-d. Chen, and Q.-x. Wu, "Sliding mode disturbance observer-based adaptive control for uncertain MIMO nonlinear systems with dead-zone," International Journal of Adaptive Control and Signal Processing, vol. 31, no. 7, pp. 1003-1018, 2017.

[19] J. Chen, J. Zhao, F. Xu, H. Hu, Q. Ai, and J. Yang, "Modeling of energy demand in the greenhouse using PSO-GA hybrid algorithms," Mathematical Problems in Engineering, vol. 2015, Article ID 871075, 6 pages, 2015.

[20] A. Ramríez-Arias, F. Rodríguez, J. L. Guzmán, and M. Berenguel, "Multiobjective hierarchical control architecture for greenhouse crop growth," Automatica, vol. 48, no. 3, pp. 490498, 2012.

[21] L. D. Albright, R. S. Gates, K. G. Arvanitis, and A. E. Drysdale, "Environmental control for plants on earth and in space," IEEE Control Systems Magazine, vol. 21, no. 5, pp. 28-47, 2001.

[22] G. D. Pasgianos, K. G. Arvanitis, P. Polycarpou, and N. Sigrimis, "A nonlinear feedback technique for greenhouse environmental control," Computers and Electronics in Agriculture, vol. 40, no. 1-3, pp. 153-177, 2003.

[23] A. Levant, "Robust Exact Differentiation via Sliding Mode Technique," Automatica, vol. 34, no. 3, pp. 379-384, 1998.

[24] N. Chatrenour, H. Razmi, and H. Doagou-Mojarrad, "Improved double integral sliding mode MPPT controller based parameter estimation for a stand-alone photovoltaic system," Energy Conversion and Management, vol. 139, pp. 97-109, 2017. 


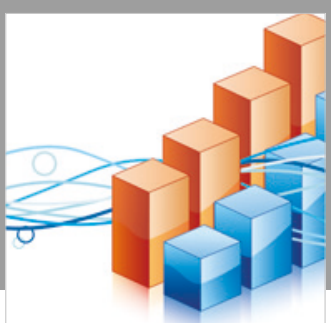

Advances in

Operations Research

\section{-n-m}
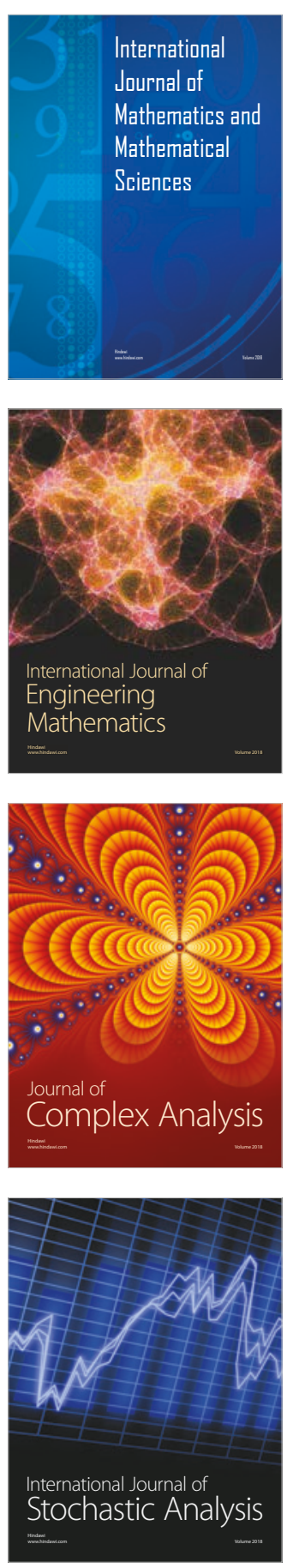
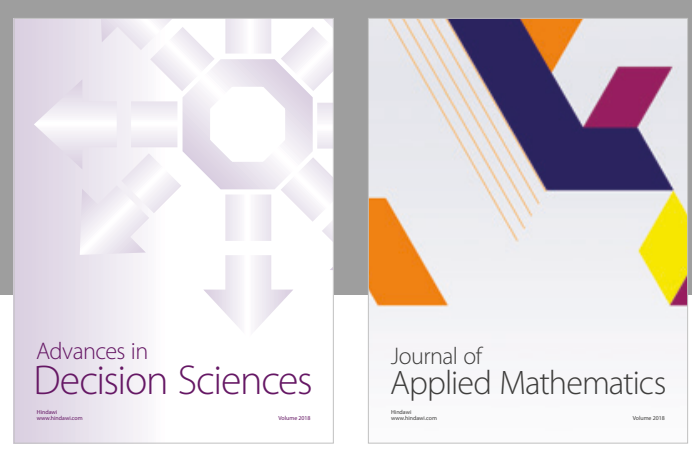

Journal of

Applied Mathematics
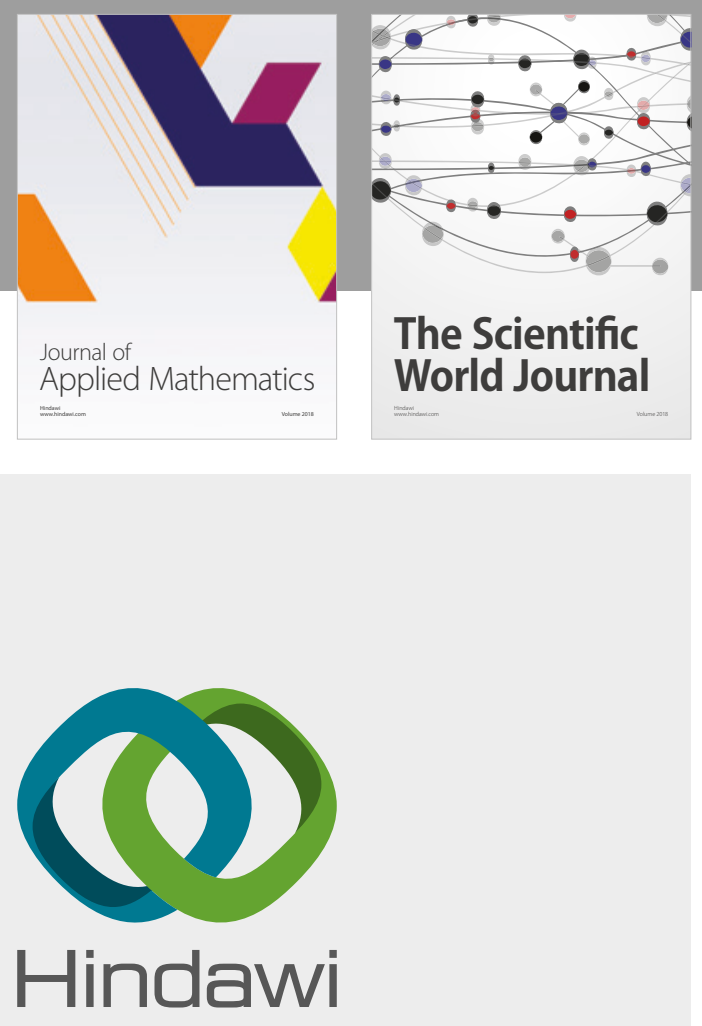

Submit your manuscripts at

www.hindawi.com

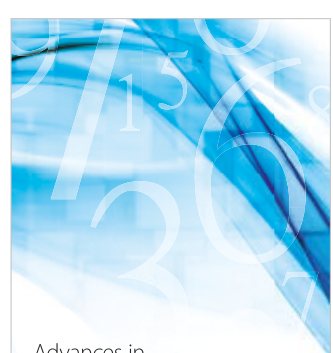

Advances in
Numerical Analysis
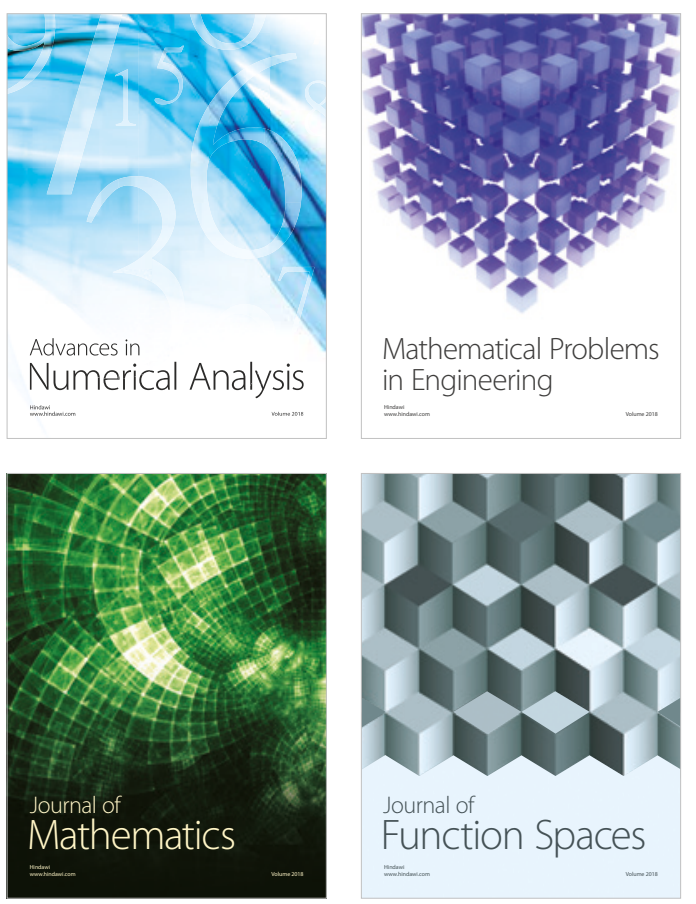

Mathematical Problems in Engineering

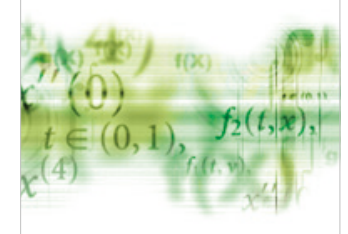

International Journal of

Differential Equations

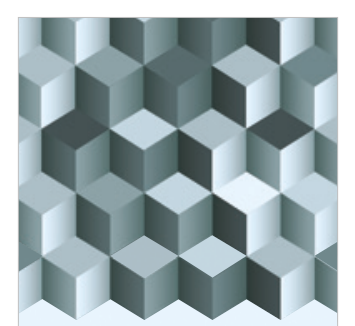

Journal of

Function Spaces

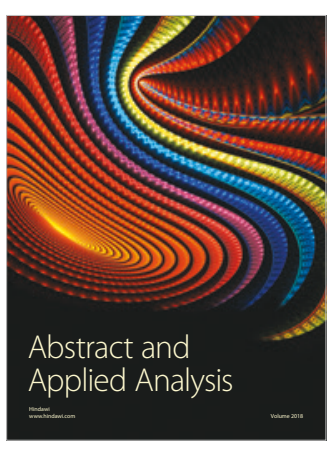

The Scientific

World Journal

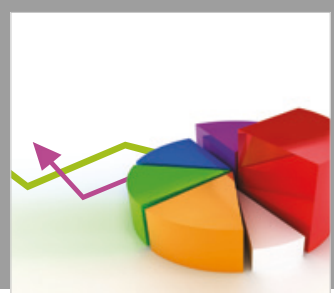

Journal of

Probability and Statistics
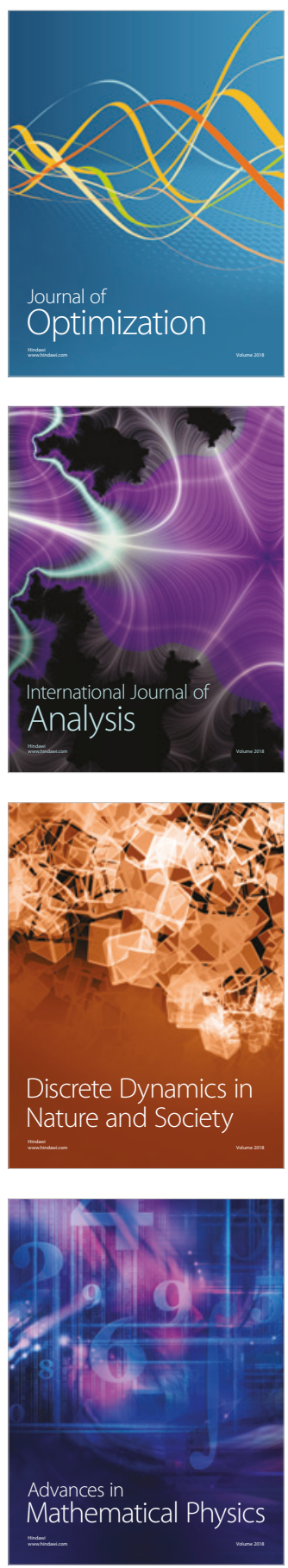